NASA Technical Memorandum 102798

\title{
Direct Inversion of Rigid-Body Rotational Dynamics
}

Ralph Bach and Russell Paielli

May 1990

(NASA-TM-102798) DIRECT INVERSION OF RIGID-BOOY ROTATIONAL OYNAMICS (NASA) 16 p
CSCL OIC

N90-21773 $\begin{array}{ll}\text { Unclas } \\ 63 / 08 & 0280231\end{array}$ 
NASA Technical Memorandum 102798

\section{Direct Inversion of Rigid-Body Rotational Dynamics}

Ralph Bach and Russell Paielli, Ames Research Center, Moffett Field, California

May 1990

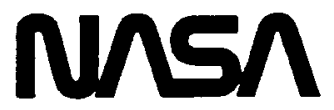

National Aeronautics and

Space Administration 


\section{SUMMARY}

In this paper, the global linearization (inversion) of rigid-body rotational dynamics is reviewed and representations in terms of quaternions and direction cosines are compared. Certain properties common to quaternions and direction cosines that make their use preferable to Euler angles and that simplify the inversion procedure are described. Applications of the inversion procedure for state estimation and attitude control are discussed. To avoid complexities caused by aerodynamics, an example of direct inversion for linear feedback control of spacecraft attitude is given.

\section{INTRODUCTION}

The problem of linearization of nonlinear systems by inversion has received much attention in the past several years, especially in aerospace and robotic control applications (refs. 1-5). The inversion procedure has also been shown to be advantageous for use in state-estimation applications (refs. 6 and 7). It is unnecessary to elaborate upon the desirability of using linear methodolgy for control or estimation of a nonlinear system. Ideally, the linearization results in an exact cancellation of nonlinear terms; it can be thought of as a nonlinear version of pole-zero cancellation. For representations of rigid-body dynamics without aerodynamic effects, the inversion is achieved by applying coordinate transformations.

This paper addresses the inversion of rigid-body rotational dynamics and illustrates its application for attitude formulations in terms of direction cosines and quaternions. (It is well-known that an Euler-angle formulation, although readily invertible, is unsuitable for representing large-angle maneuvers.) The intent of the paper is partly tutorial; it reveals the similarities in structure and the differences in performance for the two attitude formulations considered. However, some apparently little-known properties shared by the quaternion and direction-cosine formulations are described that lead to the realization of a new model-follower control system that exhibits exact linear attitude-error dynamics.

The paper consists of four major sections. In the first section, a way of expressing the direction-cosine kinematic equation is described that simplifies its inversion. An application for flight-data state estimation is suggested, and an example of spacecraft attitude control is given. A parallel treatment of a quaternion attitude formulation is provided in the following section, with the same applications. In the third section, a method is derived to realize exact linear attitude-error dynamics. Finally, in a separate section, simulation results for the control-system examples are presented and the actual performance of the formulations is compared and correlated with the error analyses made in previous sections. 


\section{DIRECTION-COSINE FORMULATION}

Consider first the matrix that rotates a vector from an inertial frame I into a body frame B with the usual Euler-angle sequence $(\psi, \theta, \varphi)$ given by (ref. 8 )

$$
\mathrm{C}_{\mathrm{BI}}=\left[\begin{array}{ccc}
\cos \theta \cos \psi & \cos \theta \sin \psi & -\sin \theta \\
\sin \varphi \sin \theta \cos \psi & \sin \varphi \sin \theta \sin \psi & \sin \varphi \cos \theta \\
-\cos \varphi \sin \psi & +\cos \varphi \cos \psi & \\
\cos \varphi \sin \theta \cos \psi & \cos \varphi \sin \theta \sin \psi & \cos \varphi \cos \theta \\
+\sin \varphi \sin \psi & -\sin \varphi \cos \psi &
\end{array}\right]
$$

Now, to eliminate dependence on trigonometric functions of the Euler angles, the rotation matrix can be formulated in terms of its column vectors as

$$
C_{B I}=\left[c_{1} c_{2} c_{3}\right], \quad c_{j}=\left[c_{1 j} c_{2 j} c_{3 j}\right]^{t}, j=1,2,3
$$

where the $\left\{c_{i j}\right\}$ are the direction cosines.

The usual way to express the kinematic equation that describes the evolution of the direction cosines when the body is rotating with angular velocity $\omega$ with respect to the inertial frame is

$$
\dot{\mathrm{C}}_{\mathrm{BI}}=-(\omega \mathrm{x}) \mathrm{C}_{\mathrm{BI}}, \quad \omega=(\mathrm{pqr})^{\mathrm{t}}
$$

where the cross-product operator is a matrix defined as

$$
(\omega x)=\left[\begin{array}{rrr}
0 & -r & q \\
r & 0 & -p \\
-q & p & 0
\end{array}\right]
$$

In this case, the objective of inversion is to determine if there exists some transformation of variables that will permit $\omega$ and its derivative to be expressed in terms of the direction-cosine elements and their derivatives, such that the state equations are linear. Although the orthogonality property of $C_{B I}$ can be used to solve equation 3 for the matrix $(\omega x)$, it is convenient to express the direction-cosine elements as the $9 \times 1$ vector

$$
c=\left[c_{1}^{t} c_{2}^{t} c_{3}^{t}\right]^{t}
$$

and use the equivalent kinematic equation 


$$
\dot{c}=C \omega, C=\left[\begin{array}{l}
c_{1} x \\
c_{2} x \\
c_{3} x
\end{array}\right]
$$

to demonstrate the inversion procedure. First, multiply both sides of equation 6 by $\mathrm{Ct}^{\mathrm{t}}$, and use the identity

$$
C^{t} \mathrm{C}=2 \mathrm{I}_{3}
$$

to obtain the expression for angular velocity

$$
\omega=C^{t} \dot{c} / 2
$$

Next, differentiate both sides of equation 8 , and use the identities

$$
\mathrm{C}^{\mathrm{t}} \mathrm{c}=0, \quad \dot{\mathrm{C}}^{\mathrm{t}} \dot{\mathrm{c}}=0
$$

to obtain the expression for angular acceleration

$$
\dot{\omega}=C^{t} \ddot{c} / 2
$$

The diagram in figure 1(a) serves to illustrate how these results might be used in a stateestimation application for flight-data filtering or smoothing. In the estimation problem, the input $\mathrm{w}$ and the initial conditions (in the smoothing case) are provided by the estimation algorithm. Note that the state equations are linear (represented by decoupled integrator strings), and that nonlinearities appear only in the expressions for the outputs (Euler angles, angular rates, and accelerations). The Euler angles are determined in the block labeled $A_{c}$ from the relations

$$
\varphi=\tan ^{-1}\left(c_{23} / c_{33}\right), \quad \theta=-\sin ^{-1} c_{13}, \quad \psi=\tan ^{-1}\left(c_{12} / c_{11}\right)
$$

A formulation like that shown in figure 1(a) significantly improves the efficiency of the stateestimation algorithms (refs. 6 and 7).

Note that while $\omega$ and $\dot{\omega}$ are expressed in terms of $c, \dot{c}$, and $\ddot{c}$, the system is truly invertible only if the constraints among the $\left\{c_{i j}\right\}$ can be maintained. In practice, only two columns (six elements) of the rotation matrix must be determined by solving differential equations - the third column of the matrix can be determined from

$$
c_{3}=c_{1} \times c_{2}
$$

while the remaining orthogonality constraints

$$
c_{1}^{t} c_{1}=1, c_{2}^{t} c_{2}=1, c_{1}^{t} c_{2}=0
$$


can be realized by adding to the output list the quantities $c_{1}^{t} c_{1}, c_{2}^{t} c_{2}, c_{1}^{t} c_{2}$, and including with the measurement set three corresponding pseudo measurement records containing 1,1 , and 0 , respectively.

\section{Representation of Attitude Error}

In attitude estimation or control applications, there is a need for a practical definition of attitude error. In terms of direction cosines, a useful error measure is given by expression

$$
\varepsilon_{c}=C^{t} c^{*} / 2
$$

where $c^{*}$ is a commanded (or estimated) attitude. The identity of equation 9 indicates that when $c=c^{*}$, the error is zero. Now, it is easy to show that

$$
\varepsilon_{c}=-\left[\begin{array}{l}
c_{23}-c_{32} \\
c_{31}-c_{13} \\
c_{12}-c_{21}
\end{array}\right] / 2
$$

where the $\left\{c_{i j}\right\}$ are elements of the matrix

$$
\mathrm{C}_{\mathrm{BB}^{*}}=\mathrm{C}_{\mathrm{BI}} \mathrm{C}_{\mathrm{IB}^{*}}
$$

which represents the rotation from the commanded axes $\left(\mathrm{B}^{*}\right)$ to the vehicle body axes $(\mathrm{B})$. Meyer (ref. 9) has shown that the measure of equation 14 is a parameterization of a single rotation about the

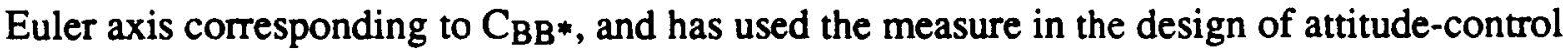
systems.

\section{A Control Example}

To illustrate, suppose that it is desired to synthesize a control law for spacecraft attitude in terms of direction cosines. Let $\tau$ denote an applied external torque, which is to be computed from

$$
\tau=\mathrm{J} \dot{\omega}+\omega \times \mathrm{J} \omega
$$

where $J$ is the spacecraft inertia matrix. A linear control law that will produce the torque necessary for a stable response is given by

$$
\dot{\omega}=\mathrm{a}_{0} \varepsilon_{\mathrm{c}}-\mathrm{a}_{1} \omega
$$

where $a_{0}, a_{1}$ are scalars adjusted to achieve reasonable transient performance. Note that although the control law is linear, the attitude-error dynamics are nonlinear. This control law has been used by Meyer (refs. 1 and 9), who has shown it to be asymptotically stable for $a_{0}, a_{1}>0$. 
The resulting attitude control system is shown in the block diagram of figure 2(a). It is readily verified that for an initial attitude of $\varphi=\theta=\psi=0$, the initial errors are given by

$$
\varepsilon_{\mathrm{c}}=\left[\begin{array}{c}
\sin \varphi^{*} \\
0 \\
0
\end{array}\right], \varepsilon_{\mathrm{c}}=\left[\begin{array}{c}
0 \\
\sin \theta^{*} \\
0
\end{array}\right], \varepsilon_{\mathrm{c}}=\left[\begin{array}{c}
0 \\
0 \\
\sin \psi^{*}
\end{array}\right]
$$

for single-axis rotation commands of $\varphi^{*}, \theta^{*}$, and $\psi^{*}$, respectively. Hence, the control system should follow any single-axis rotation commands within the range $\pm 180^{\circ}$.

\section{QUATERNION FORMULATION}

Euler's theorem states that any orientation of a body frame B with respect to an inertial frame I can be expressed as a single rotation about some axis through their common origin. That orientation can be represented by a set of four parameters called quaternions. The quaternion vector is (ref. 10)

$$
e=\left[e_{1} e_{2} e_{3} e_{4}\right]^{t}
$$

where

$$
e_{i}=d_{i} \sin \alpha / 2, i=1,2,3, \quad e_{4}=\cos \alpha / 2
$$

In equation $21, \alpha$ is the rotation about the Euler axis, and the $\left(d_{i}\right)$ are the direction cosines of the Euler axis with respect to the inertial frame. There is only one constraint among the quaternion elements:

$$
e^{t} e=1
$$

It should be mentioned that the rotation matrix of equation 1 can also be expressed in terms of the quaternion elements as (ref. 10)

$$
C_{B I}=\left[\begin{array}{lll}
1-2\left(e_{2}^{2}+e_{3}^{2}\right) & 2\left(e_{1} e_{2}+e_{3} e_{4}\right) & 2\left(e_{1} e_{3}-e_{2} e_{4}\right) \\
2\left(e_{1} e_{2}-e_{3} e_{4}\right) & 1-2\left(e_{1}^{2}+e_{3}^{2}\right) & 2\left(e_{2} e_{3}+e_{1} e_{4}\right) \\
2\left(e_{1} e_{3}+e_{2} e_{4}\right) & 2\left(e_{2} e_{3}-e_{1} e_{4}\right) & 1-2\left(e_{1}^{2}+e_{2}^{2}\right)
\end{array}\right]
$$

The attitude dynamics in a quaternion formulation are conveniently described by the kinematic equation 


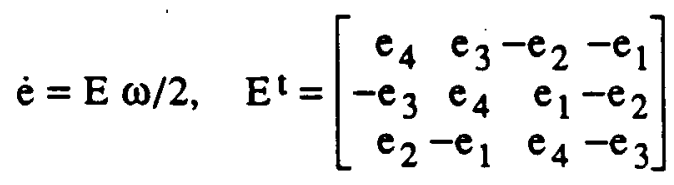

It is of interest to observe (and perhaps it should be anticipated) that a set of identities similar to those given in equations 7 and 9 also exists for a quaternion attitude formulation. The quatemion set is

$$
\mathrm{E}^{\mathrm{t}} \mathrm{E}=\mathrm{I}_{3}, \mathrm{E}^{\mathrm{t}} \mathrm{e}=0, \dot{\mathrm{E}}^{\mathrm{t}} \dot{\mathrm{e}}=0
$$

To see how this formulation may be inverted, note the identities above and proceed as in the previous inversion to obtain the angular velocity and acceleration, which in this case are given by

$$
\omega=2 E^{t} \dot{e}, \quad \dot{\omega}=2 E^{t} \ddot{e}
$$

The corresponding state-estimation model for the quaternion attitude formulation is shown in figure 1(b). To realize the magnitude constraint of equation 22 , the left side can be added to the output list, and a measurement record of 1 's added to the measurement set. Here there are only four variables to be determined by solving differential equations, with one additional measurement, compared to six variables with three additional measurements in the direction-cosine formulation. The question of which formulation would be preferable for a particular filtering or smoothing application, however, could depend on how often the rotation matrix had to be calculated. Implementation of the models shown in figures 1 (a) and (b) is a subject of ongoing research.

\section{A Control Example}

A useful measure of attitude error in a quatemion formulation is given by

$$
\varepsilon_{\mathrm{e}}=2 \mathrm{E}^{\mathrm{t}} \mathrm{e}^{*}
$$

where $e^{*}$ is the attitude command. The identify of equation 25 indicates that when $e=e^{*}$, the error is zero. The special properties of this measure lead to the exact linearization procedure to be discussed in the next section. First, however, consider its use with a feedback control system like that shown in figure 2(a), but based on quaternions. Such a system has been described by Wie et al. (ref. 11), and is included here as an example to facilitate a comparison of the direction-cosine and quaternion formulations. It also uses a linear control law

$$
\dot{\omega}=\mathrm{a}_{0} \varepsilon_{\mathrm{e}}-\mathrm{a}_{1} \omega
$$

where as before, $a_{0}, a_{1}$ are scalars. Wie has shown that this nonlinear system is asymptotically stable for $a_{0}, a_{1}>0$.

A block diagram for the quaternion control system is shown in figure $2(\mathrm{~b})$. In this case, for an initial body attitude of $\varphi=\theta=\psi=0$, the attitude error becomes 


$$
\varepsilon_{\mathrm{e}}=2\left[\begin{array}{l}
e_{1} \\
e_{2} \\
e_{3}
\end{array}\right]^{*}=2 \sin \left(\alpha^{*} / 2\right)\left[\begin{array}{l}
d_{1} \\
d_{2} \\
d_{3}
\end{array}\right]^{*}
$$

For single-axis rotation commands of $\varphi^{*}, \theta^{*}, \psi^{*}$ the initial errors are given by

$$
\varepsilon_{\mathrm{e}}=2 \sin \left(\varphi^{*} / 2\right)\left[\begin{array}{l}
1 \\
0 \\
0
\end{array}\right], 2 \sin (\theta * / 2)\left[\begin{array}{l}
0 \\
1 \\
0
\end{array}\right], 2 \sin \left(\psi^{*} / 2\right)\left[\begin{array}{l}
0 \\
0 \\
1
\end{array}\right]
$$

which implies that the quaternion control system can follow any rotation command within $\pm 360^{\circ}$.

\section{An Exact Linearization}

The direction-cosine and quaternion attitude-control examples considered in previous sections exhibit nonlinear dynamics for large errors. In this section, some properties of the quaternion error measure are described that make it possible to realize an exact linearization of the error dynamics for either formulation. The results reported here represent an extension of the work of Dwyer (ref. 3).

To begin, define a vector $e$ (with elements $e_{1}, e_{2}, e_{3}$ ), and a $3 \times 3$ matrix $E$ as

$$
e=\mathrm{E}^{* \mathrm{t}} \mathrm{e}, \quad E=\mathrm{E}^{* \mathrm{t}} \mathrm{E}
$$

where $E^{*}$ is the matrix in the quaternion kinematics of equation 24 with $e$, the actual body attitude, replaced by $\mathrm{e}^{*}$, the commanded attitude. Note that $e$ is proportional to the quaternion attitude-error measure of equation 27. From the definitions given in equation 31 it follows that

$$
E=\left[\begin{array}{ccc}
\lambda & -e_{3} & e_{2} \\
e_{3} & \lambda & -e_{1} \\
-e_{2} & e_{1} & \lambda
\end{array}\right], \quad \lambda=\mathrm{e}^{\mathrm{t}} \mathrm{e}^{*}
$$

It should be apparent from equations 31 and 32 that when $\mathrm{e}=\mathrm{e}^{*}$, that $e=0, \lambda=1$, and that $E$ is the unit matrix.

The derivation of the linearization procedure depends on observing that

$$
E=(e \mathrm{x})+\lambda \mathrm{I}_{3}, \quad E e=\lambda e
$$

(therefore $\lambda$ is an eigenvalue and $e$ is a corresponding eigenvector of $E$ ), and 


$$
E^{-1}=E^{t}+e e^{t / \lambda}, \quad E^{-l} E^{t}=\mathrm{C}_{\mathrm{BB}^{*}}
$$

where $\mathrm{C}_{\mathrm{BB}}$ is the attitude-error rotation matrix mentioned earlier. Now, if the error in angular rate is defined as

$$
\omega=\omega-\mathrm{C}_{\mathrm{BB}} * \omega^{*}
$$

where $\omega^{*}$ is the commanded rate, equations 24,34 , and 35 can be used to derive the error kinematic equation as

$$
\dot{e}=E \omega / 2
$$

Hence, $e$ can be thought of as making up the first three elements of an attitude-error quaternion, with $\lambda$ being the fourth quaternion element.

To complete the inversion derivation, first differentiate equation 35 and express the body coordinates of angular acceleration as

$$
\dot{\omega}=\mathrm{C}_{\mathrm{BB}^{*}} \dot{\omega}^{*}+\left(\mathrm{C}_{\mathrm{BB}^{*}} \omega^{*}\right) \times \omega+\dot{\omega}
$$

where $\dot{\omega}^{*}$ is the commanded acceleration, and then differentiate equation 36 and solve for $\dot{\omega}$ as the expression

$$
\dot{\omega}=2 E^{-1}\left[\ddot{e}-\left(\omega^{\mathrm{t}} \omega / 4\right)\right]
$$

Finally, to achieve an exact linear attitude-error response corresponding to

$$
\ddot{e}+\mathrm{a}_{1} \dot{e}+\mathrm{a}_{0} e=0 \quad \text { (or } \ddot{\varepsilon}_{\mathrm{e}}+\mathrm{a}_{1} \dot{\varepsilon}_{\mathrm{e}}+\mathrm{a}_{0} \varepsilon_{\mathrm{e}}=0 \text { ) }
$$

substitute for $\ddot{e}$ in equation 38 and use equation 34 to obtain the feedback control law

$$
\dot{\omega}=\mathrm{a} \varepsilon_{\mathrm{e}}-\mathrm{a}_{1} \omega, \mathrm{a}=\left[\mathrm{a}_{0}-\left(\omega^{\mathrm{t}} \omega / 4\right)\right] \lambda
$$

With the control law of equation 40 , the quaternion system exhibits an exact linear error response for large errors. Note, however, that $\lambda=0$ for attitude errors of $\pm 180^{\circ}$. Hence, linearity is achieved at the expense of angular range for the quaternion system. An attitude-control system implemented with direction cosines can also be designed to exhibit the same linear response, since it can be shown that

$$
\varepsilon_{\mathrm{e}}=\varepsilon_{\mathrm{c}} / \lambda, \quad \lambda^{2}=\left(1+\mathrm{c}^{\mathrm{t}} \mathrm{c}^{*}\right) / 4
$$

The expression in equation 41 that relates $\lambda$ to the trace of the error-rotation matrix is an identity (ref. 10). Note that $\lambda^{2}=1$ when $c=c^{*}$. The corresponding control law for the direction-cosine system is given by 


$$
\dot{\omega}=\mathrm{a} \varepsilon_{c}-\mathrm{a}_{1} \omega, \quad \mathrm{a}=\left[\mathrm{a}_{0}-\left(\omega^{\mathrm{t}} \omega / 4\right)\right] / \lambda^{2}
$$

The linearization procedure described here is applicable to model-follower feedback control systems (ref. 1). Its implementation for practical applications is a subject of current interest. However, to linearize the simple attitude-control systems shown in figure 2 , where the commanded rate and acceleration are zero, simply replace an with the appropriate time-varying gain a (from eqs. 40 . or 42 , with $\omega=\omega$ ). The quaternion version of the exact linear-error system was used to obtain the simulation results presented in the next section.

\section{SIMULATION RESULTS}

For the attitude-control simulations prepared for this section, it was assumed that the spacecraft inertia matrix was known exactly. The error analyses given in previous sections indicate that the two linear feedback attitude-control system formulations will exhibit similar (nearly linear) performance for small-angle commands. Therefore, the system gains for those simulations as well as the exact linear-error simulation were adjusted to obtain critical damping, with $a_{0}=4, a_{1}=4$ (poles at $-2 \mathrm{rad} / \mathrm{sec}$ ). The commands were given in terms of Euler angles, which were then converted to corresponding commands $\mathrm{c}^{*}$ or $\mathrm{e}^{*}$.

Recall that the direction-cosine system of figure 2 (a) is limited to following commands in the range $\pm 180^{\circ}$, while the quaternion system of figure 2(b) can follow commands in the range $\pm 360^{\circ}$. Although these facts are well known to industry practitioners, they are worthwhile emphasizing here. The performance of the two systems for roll-angle commands of $270^{\circ}$ is shown in figure 3(a). The important difference in the direction-cosine and quaternion formulations, however, is that the latter exhibits a maximum attitude error amplitude for commands of $\pm 180^{\circ}$, for which the former system exhibits no error at all. The quaternion system should, therefore, be more robust in responding to large-angle commands. This property is demonstrated by the responses of the two systems for roll commands of $170^{\circ}$ shown in figure $3(b)$.

The exact linear-error simulation was run for a command of $-90^{\circ}$ in both yaw and pitch, which corresponds to an initial condition for $e_{1}, e_{2}, e_{3}$ and $\lambda$ of 0.5 . The results are shown in figure 4. Notice that the responses for the $\left\{e_{i}\right\}$ are identical, and different from $\lambda$ ( $\lambda$ must satisfy the quaternion magnitude constraint). The responses of all systems to roll commands of $10^{\circ}$, shown in figure 5(a), are almost identical, as predicted. Finally, the responses of all three simulations to rollangle commands of $170^{\circ}$ are shown for comparison in figure 5(b). The exact linear-error simulation is seen to exhibit the most robust performance, as should be expected. Note that although the error quatermion elements of equation 31 exhibit the desired linear response to a command, the attitude behavior in an Euler-angle sense is nonlinear as determined by the relations defined in equation 11 . 


\section{CONCLUDING REMARKS}

This paper has outlined some useful properties of direction-cosine and quaternion formulations that simplify the linearization of rigid-body rotational dynamics. Applications of the inversion procedure have been given for both estimation and control examples. Error analyses and simulation results for spacecraft attitude-control systems have been presented that demonstrate the more robust performance obtainable from an exact linear-error formulation over that obtained from either direction-cosine and quartemion formulations with simple linear feedback control laws.

\section{REFERENCES}

1 Meyer, G.; and Cicolani, L: "A Formal Structure for Advanced Automatic Flight-Control Systems," NASA TN-D-7940, 1975.

2. Hunt, L. R.; Su, R.; and Meyer, G.: "Global Transformations of Nonlinear Systems," IEEE Trans. Automat. Contr., vol. AC-28, 1983, pp. 24-31.

3. Dwyer, T. A. W., III: "Exact Nonlinear Control of Large Angle Rotational Maneuvers," IEEE Trans. Automat. Contr., vol. AC-29, Sept. 1984, pp. 769-774.

4. Craig, J. J.: Introduction to Robotics, Chap. 8, Addison Wesley, Reading, MA, 1986.

5. Hauser, J.; Sastry, S.; and Meyer, G.: "Nonlinear Controller Design for Flight Control Systems," Memorandum UCB/ERL M88/76, UC Berkeley, Dec. 1988.

6. Bach, R. E., Jr.: "A Mathematical Model for Efficient Estimation of Aircraft Motions," Sixth IFAC Symposium on Identification and Estimation, June 1982.

7. Bach, R. E., Jr.: "Applications of State Estimation in Aircraft Flight-Data Analysis," ALAA J. Aircraft, vol. 22, no. 7, 1985, pp. 547-554.

8. Etkin, B.: Dynamics of Atmospheric Flight, Chap. 4, John Wiley, New York, NY, 1972.

9. Meyer, G.: "On the Use of Euler's Theorem on Rotations for the Synthesis of Attitude Control Systems," NASA TN-D-3643, 1966.

10. Hughes, P. C.: Spacecraft Attitude Dynamics, Chap. 2, John Wiley, New York, NY, 1986.

11. Wie, B.; Weiss, H.; and Arapostathis, A.: "Quaternion Feedback Regulator for Spacecraft Eigenaxis Rotations," AIAA J. Guidance Cont., vol. 12, no. 3, 1989, pp. 375-380. 

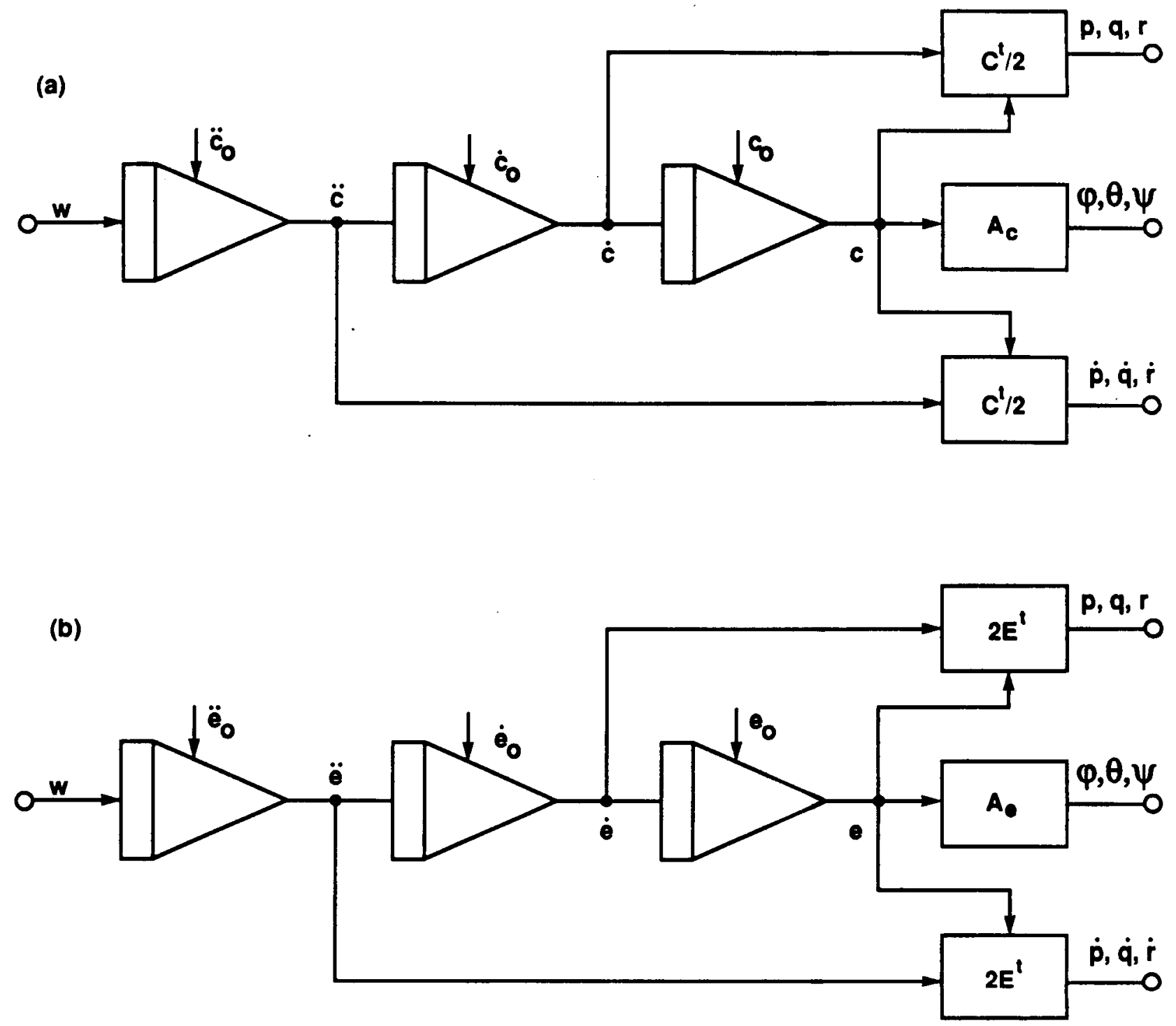

Figure 1. Attitude estimation models for flight-data filtering or smoothing applications. a) Directioncosine formulation; b) quaternion formulation. 

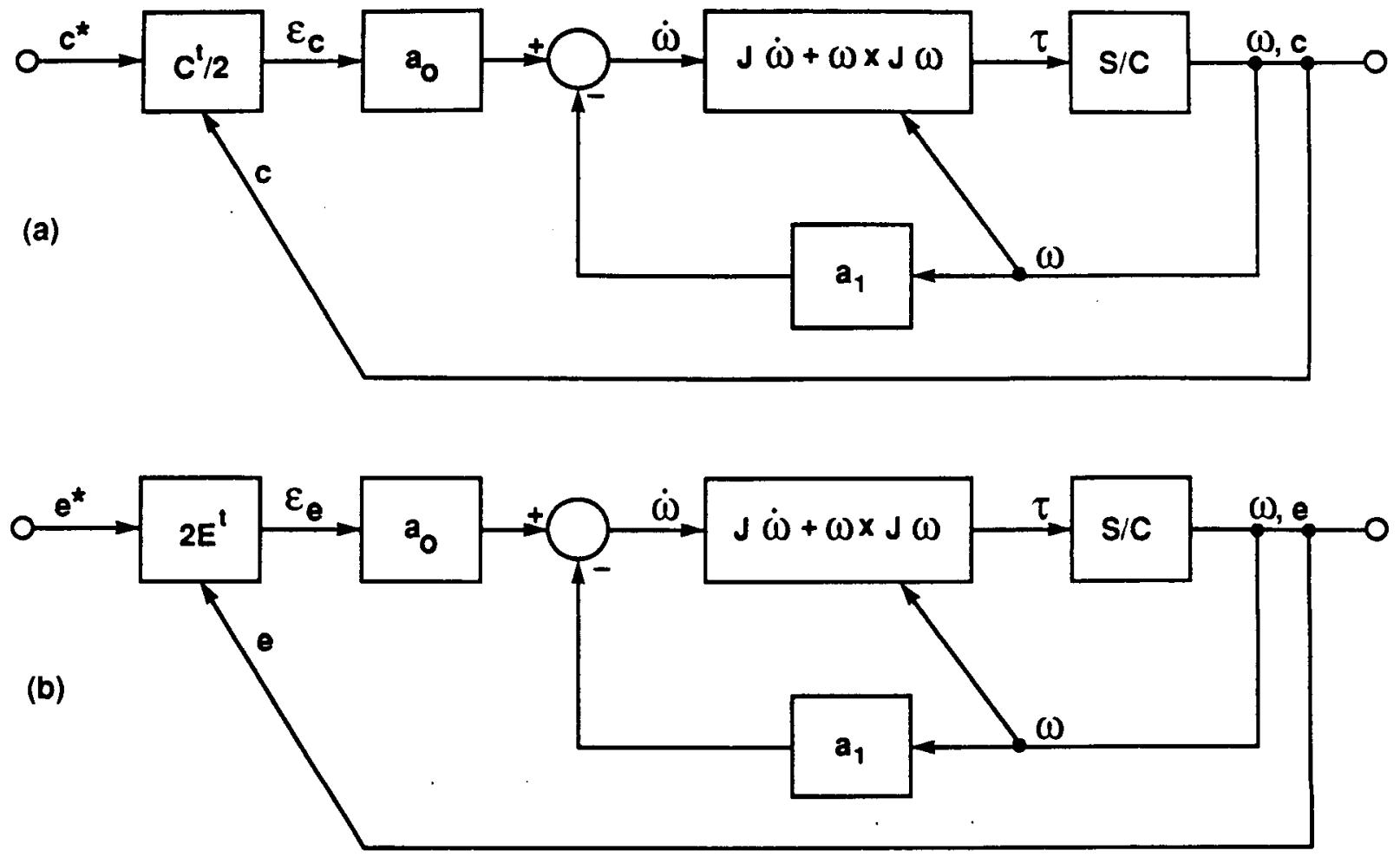

Figure 2. Spacecraft attitude control systems. a) Direction-cosine formulation; b) quaternion formulation.
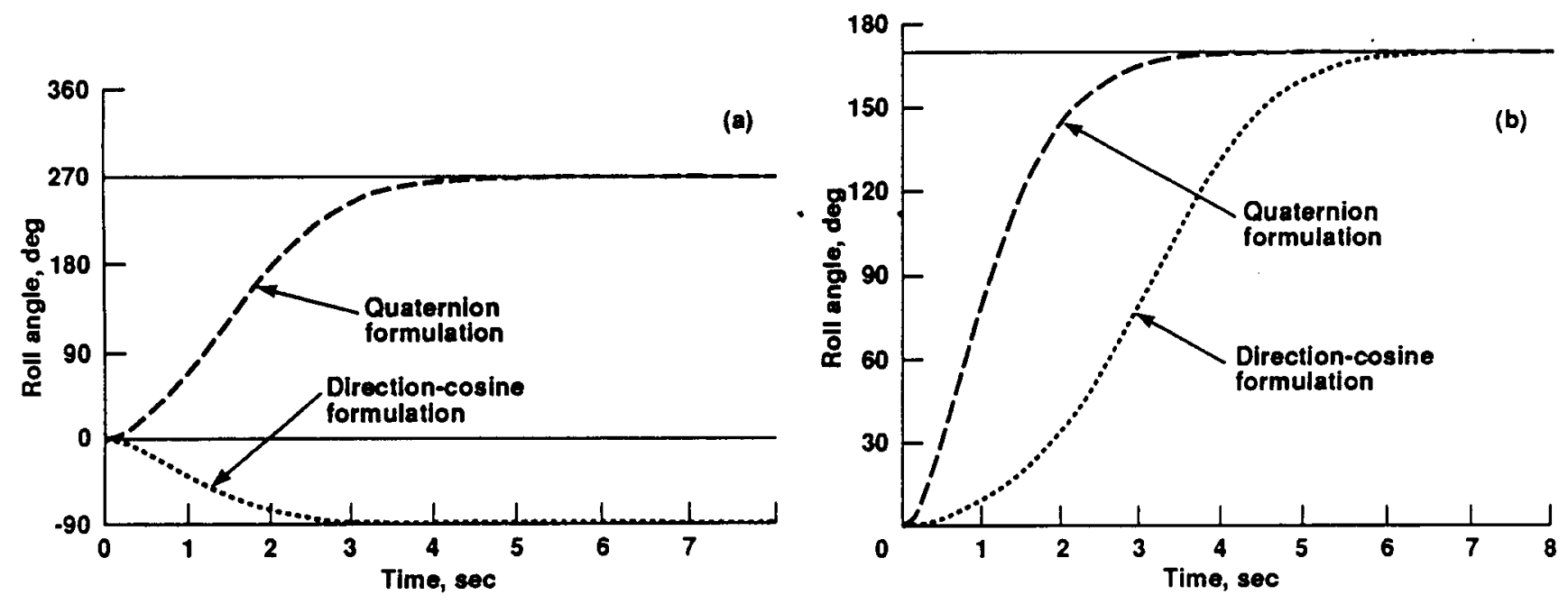

Figure 3. Response of direction-cosine and quaternion attitude-control simulations to a) roll commands of $270^{\circ} ;$ b) roll commands of $170^{\circ}$. 


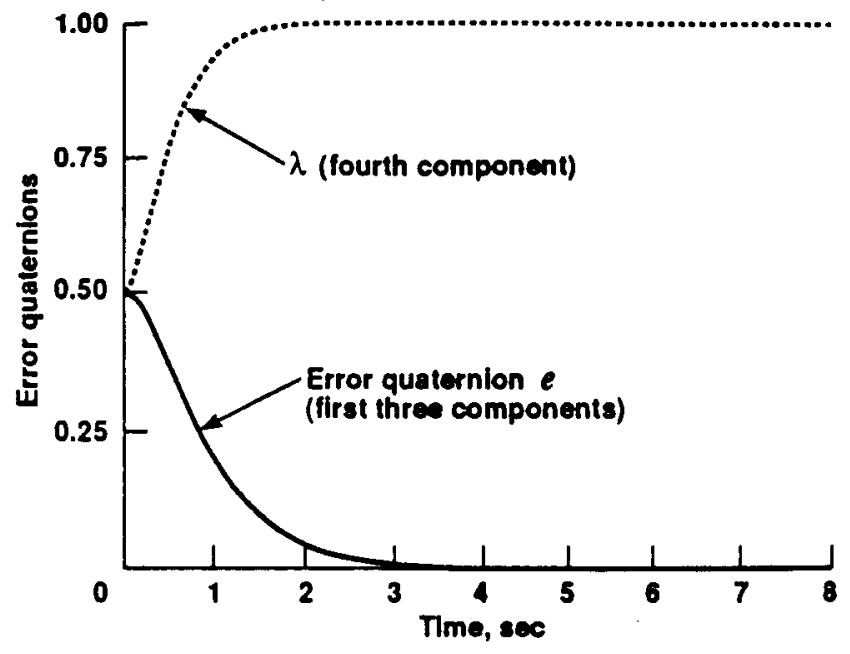

Figure 4. Response of exact linear-error quaternion simulation to commands of $-90^{\circ}$ in yaw and $-90^{\circ}$ in pitch.
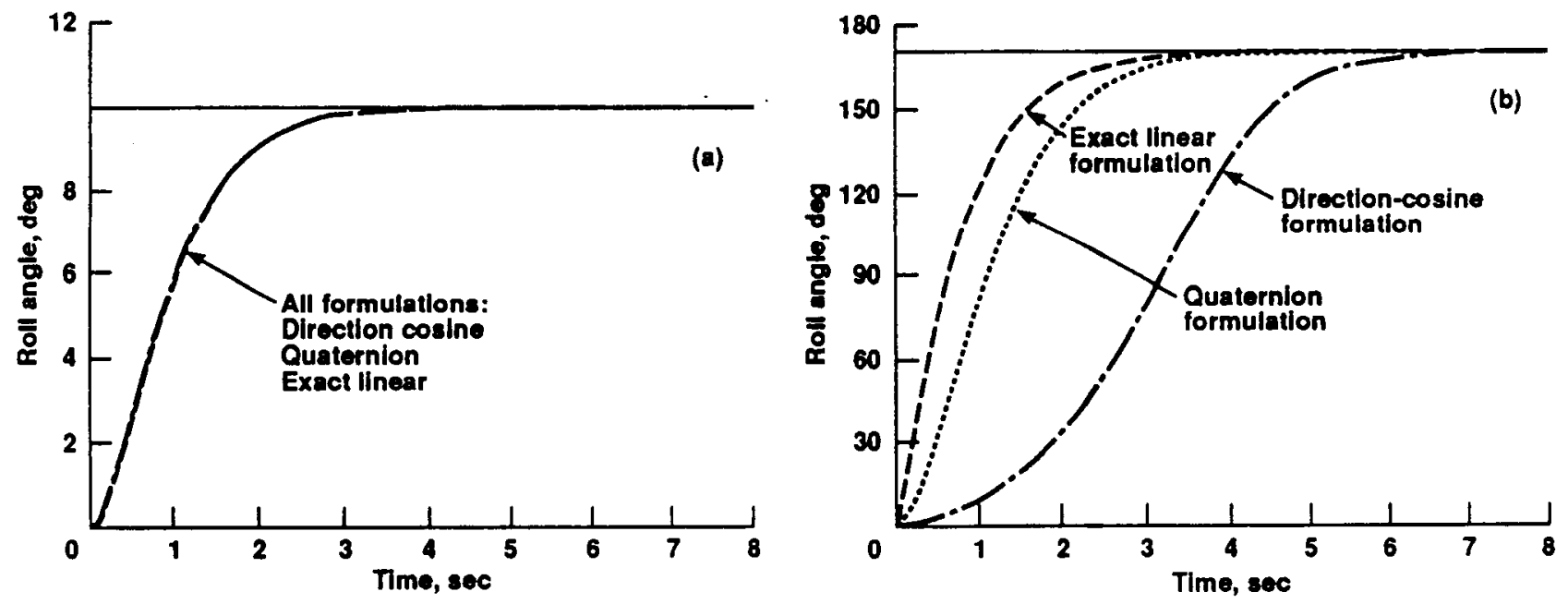

Figure 5. Response of three attitude-control simulations to a) roll-angle commands of $10^{\circ}$; b) roll-angle commands of $170^{\circ}$. 


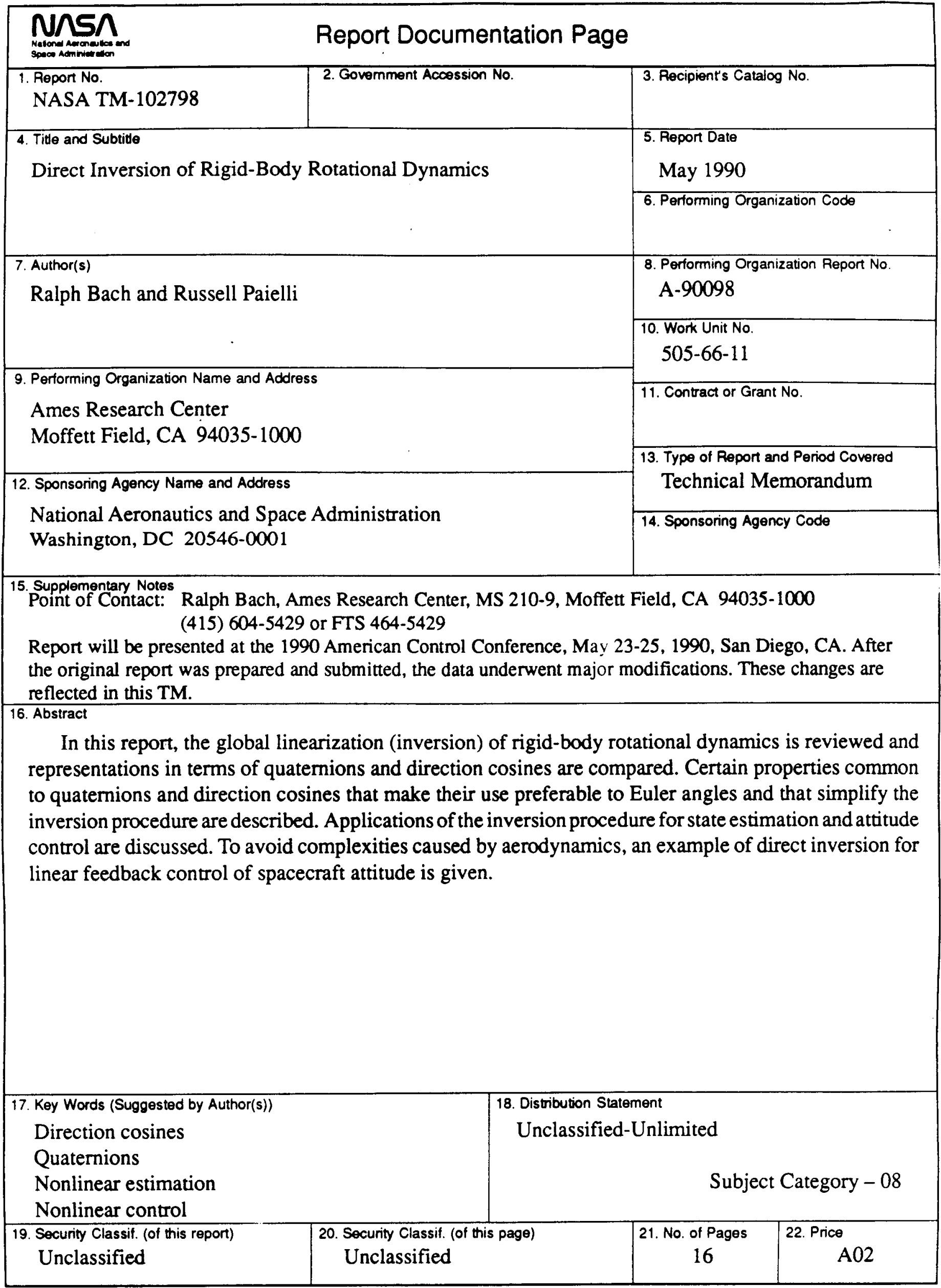

